\title{
Article \\ A Comparison of Cone Beam Computed Tomography and Periapical Digital Radiography for Evaluation of Root Canal Preparation
}

\author{
Khoa Van Pham
}

Citation: Pham, K.V. A Comparison of Cone Beam Computed Tomography and Periapical Digital Radiography for Evaluation of Root Canal

Preparation. Appl. Sci. 2021, 11, 6599. https://doi.org/10.3390/app11146599

Academic Editor: Luca Testarelli

Received: 24 June 2021

Accepted: 16 July 2021

Published: 18 July 2021

Publisher's Note: MDPI stays neutral with regard to jurisdictional claims in published maps and institutional affiliations.

Copyright: (C) 2021 by the author. Licensee MDPI, Basel, Switzerland. This article is an open access article distributed under the terms and conditions of the Creative Commons Attribution (CC BY) license (https:// creativecommons.org/licenses/by/ $4.0 /)$
Department of Operative Dentistry and Endodontics, Faculty of Odonto-Stomatology, University of Medicine and Pharmacy at Ho Chi Minh City, Ho Chi Minh City 700000, Vietnam; khoapv@ump.edu.vn; Tel.: +84-7720-00300

\begin{abstract}
The aim of the present study is to compare cone beam computed tomography and periapical digital radiography for the evaluation of root canal preparation. Nine extracted human molars were used in this study. Following access cavity preparation, mesio-buccal roots of maxillary and mesial roots of mandibular molars were prepared and the remaining roots were cut off. Three amalgam cavities were prepared on the coronal part of the teeth and were filled with amalgam to be used as landmarks. Specimens were scanned using cone-beam computed tomography and periapical digital radiograph images were obtained before and after root canal preparation. WaveOne Gold Primary was used for root canal preparation to full working length. Specimens were then scanned using $\mathrm{CBCT}$ and a periapical radiograph for the after-instrumentation images. The transportation and centering ratio were measured and calculated on the CBCT and periapical radiographic images. The Bland-Altman method was used for detecting the bias in the evaluation of agreement between the two methods' measurements. There was agreement between the two methods' measurements using CBCT scans and periapical digital radiographic images in the evaluation of transportation and centering ratio parameters. The two methods could be used interchangeably in measurements of transportation and calculating the centering ratio.
\end{abstract}

Keywords: cone-beam computed tomography; periapical digital radiograph; root canal preparation; reciprocating; transportation; centering ratio

\section{Introduction}

One of the most critical phases in endodontics is the root canal preparation. This is not only performed to obtain the main objectives of the stage but also to facilitate the following important obturation to gain the paramount goal for successful endodontic therapy [1]. Besides the main duties, such as the removal or cleaning of canal content, the root canal preparation should maintain the original canal's shape and pathway, which is assessed by certain measurements [2]. The evaluation of root canal geometry on extracted human teeth before and after endodontic instrumentation is popular for canal transportation or centering ability in endodontics [3]. This assessment is commonly used for the technique, instrument, or other mechanical intervention in root canal preparation for its effectiveness, advantages and capability [4]. From the initial canal impression utilization for endodontic preparation assessment, many methods have been developed for this task along with the development of technology [4]. With the method of Bramante, the tooth is cross sectioned to evaluate the before and after instrumentation root canal perimeter at an acceptable certain precise level, but in an invasive way [3]. In search of excellent methods of evaluation, a non-invasive way is preferred, and radiographical imaging is prospective modality [4]. From the beginning of the use of this method, mainly in two-dimensional measurements with a periapical radiograph, it has been incessantly improved, gaining the ability for three-dimensional investigation using cone beam computed tomography (CBCT) or microCBCT [4]. Although the micro-CBCT is the gold standard nowadays, the accessibility of this 
has advanced. It is confined to investigation facilities in developing countries, regardless of the certain shortcomings of the time-consuming or ex vivo only methods [3]. The CBCT was used in the comparisons of root canal preparation using different techniques [1], or in working length determination [5-8]. When using the CBCT, one of the most important parameters is the voxel size [6]. The smaller the voxel size of the scanning image, the more accurate the measurement [6]. However, the more precise the dimension, the more radiation the patient is burdened with, resulting in the limitation of CBCT manipulation in routine endodontic therapy $[6,9,10]$. The measurement of dimensions with both periapical radiograph and $\mathrm{CBCT}$ is commonly manipulated to evaluate root canal preparation. Both modalities achieve acceptable certain recognitions at certain precise levels [4]. However, the comparison between these two methods or the correlations between them have as yet not been adequately considered, and the data are limited [11].

The aim of the present study is to compare cone beam computed tomography and periapical digital radiography for the evaluation of root canal preparation.

\section{Materials and Methods}

The present study was approved by the Research Ethics Committee of the University of Medicine and Pharmacy in Ho Chi Minh City, Vietnam, with the approval number 353/QĐ-ĐHYD-RHM. The study was performed in accordance with relevant guidelines and regulations. The study acquired intact human extracted molars obtained from many hospitals for many reasons, and informed consent was obtained from all participants. The sample size for the present study was chosen using the sample size calculation submenu of the MedCalc Statistical Software version 19 (MedCalc Software, Ostend, Belgium), resulting in a sample size of nine root canals with nine human extracted molars. Teeth were cleaned, checked under stereomicroscope (Olympus SZX16, Olympus Corp., Tokyo, Japan), and coded following the procedure of the previous study [6]. Roots other than the mesio-buccal root of the upper molars and the mesial root of the lower molars were resected. Periapical digital radiographs were obtained in both mesio-distal and bucco-lingual directions to observe the root canal systems of each molar for exclusion criteria such as previous root canal treatments, calcifications, internal resorptions, abnormal obliterations, or ramifications. The root canal curvatures were measured using the radiographs in the bucco-lingual direction following the Schneider method [12]. Teeth were then prepared for access cavities with Martin and Endo-Z burs (Dentsply Sirona, Maillefer, Ballaigues, Switzerland) under copious water spray until all canal orifices were completely exposed. The canal was checked for patency using a \#10 ISO K-file (Dentsply Sirona, Maillefer, Ballaigues, Switzerland) until the tip of the file was visible at the coronal border of the apical foramen under the stereomicroscope at a magnification of ten. The root canal length was recorded using a firm reference point on the occlusal surface, corresponding to the rubber stop position on the shaft of the K-file. The working length for each canal was affirmed by less than one millimeter from the whole canal length. Three cavities for amalgam were prepared on the coronal part of the tooth using a 169 bur (Dentsply Sirona, Maillefer, Ballaigues, Switzerland) and were then filled up with amalgam. The amalgam fillings were thoroughly polished after a period of $24 \mathrm{~h}$ for full setting. All teeth were then arranged and immersed in plastic molds with light impression silicone inside, as described in the previous study [6]. Molds with teeth were scanned for the first time using the cone beam computed tomography (CBCT) (Planmeca ProMax 3D Classic, Planmeca Oy, Helsinki, Finland) with endo mode, $90 \mathrm{kV}$, $8 \mathrm{~mA}$ or $10 \mathrm{~mA}$, field of view (FOV) $50 \mathrm{~mm} \times 50 \mathrm{~mm}$, at a voxel size of $0.075 \mathrm{~mm}$. Teeth were then removed from the plastic molds and prepared for the before instrumentation periapical digital radiographs. An apparatus made of plastic water pipeline parts (Binh Minh Plastics, Ho Chi Minh City, Vietnam) was produced to ensure that the before and after instrumentation positions of the tooth were exactly overlapped (Figure 1). All parts of the apparatus were created and modified so that the before and after instrumentation positions of the tooth had exactly overlapped each other, in both bucco-lingual and mesio-distal directions, facilitating the overlapping of the before and after instrumentation images 
for evaluation. The digital sensor (Sopix2, Sopro, La Ciotat, France) of the radiograph apparatus (X-Mind, Satelec, Acteon Group, France) was fixed on a removable plastic cap, fitted to the end of a long plastic pipeline. The middle part, containing the customized medium silicone matrix (GC Corp., Tokyo, Japan) with the tooth inside, was then inserted and kept by an edge inside the long tube. Each tooth specimen had been inserted into the silicone impressions following two directions, the first for the bucco-lingual direction and the second for the mesio-distal direction. The tooth was then removed from the silicone impressions and prepared for root canal instrumentation using WaveOne Gold (Dentsply Maillefer, Baillagues, Switzerland) of the Primary instrument with the WaveOne motor (Dentsply Maillefer, Baillagues, Switzerland), until the working length was reached. Every WaveOne Gold Primary instrument was used for only one canal. The root canal was irrigated using 3\% sodium hypochlorite (CanalPro, Coltène/Whaledent GmbH, Langenau, Germany) before and after instrumentation, and then dried using a paper cone. The teeth were then inserted back into the silicone impressions and were scanned for the second time using CBCT with the same parameters as the first time. Each tooth, after that, was inserted into its own impression as created previously, and was exposed to the periapical digital radiograph, in both directions, for images of after instrumentation. The dataset from the CBCT was processed using the formulation in the previous study, with the data being recorded from the point of $3 \mathrm{~mm}$ from the apex foramen, which was $2 \mathrm{~mm}$ from the working length [1]. For each corresponding pair of CBCT slices, eight parameters were measured and values were calculated from these parameters. $M_{b}^{c}$ represented the shortest distance from the mesial root surface to the periphery of the before instrumentation canal; $M_{a}^{c}$ represented the shortest distance from the mesial root surface to the periphery of the after instrumentation canal; $D_{b}^{c}$ represented the shortest distance from the distal root surface to the periphery of the before instrumentation canal; and $D_{a}^{c}$ represented the shortest distance from the distal root surface to the periphery of the after instrumentation canal. The difference between each pair of measurements $\left|\left(M_{b}^{c}-M_{a}^{c}\right)-\left(D_{b}^{c}-D_{a}^{c}\right)\right|$ value and the ratio between these two differences $\left|\left(M_{b}^{c}-M_{a}^{c}\right) /\left(D_{b}^{c}-D_{a}^{c}\right)\right|$ value (when the numerator was the smaller of these pairs of numbers) were the transportation and centering ratio in the mesio-distal direction, respectively. $B_{b}^{c}$ represented the shortest distance from the buccal root surface to the periphery of the before instrumentation canal; $B_{a}^{c}$ represented the shortest distance from the buccal root surface to the periphery of the after instrumentation canal; $L_{b}^{c}$ represented the shortest distance from the lingual root surface to the periphery of the before instrumentation canal; and $L_{a}^{c}$ represented the shortest distance from the lingual root surface to the periphery of the after-instrumentation canal. The difference between each pair of measurements $\left|\left(B_{b}^{c}-B_{a}^{c}\right)-\left(L_{b}^{c}-L_{a}^{c}\right)\right|$ value and the ratio between these two differences $\left|\left(B_{b}^{c}-B_{a}^{c}\right) /\left(L_{b}^{c}-L_{a}^{c}\right)\right|$ value (when the numerator was the smaller of these pairs of numbers) were the transportation and centering ratio in the bucco-lingual direction, respectively (Figure 2).

The periapical image was processed using the modified method described by a previous study [2]. The trace of the root canal outline was performed using the Sketch software for mobile phones (SamSung Galaxy Note 10, Samsung, Suwon, Korea). Data were transferred to the computer for further measurements and calculations. The Adobe Photoshop CC 2019 software (Adobe, San Jose, CA, USA) was used for overlapping the before and after instrumentation images, creating the composite image for further analysis (Figure 3). After that, the Image 1.52 (National Institutes of Health, Bethesda, Rockville, MD, USA) was used for tracing and measuring the data on the images acquired from the above step. Measurements were performed on each millimeter from the apex foramen to the level of $7 \mathrm{~mm}$ from the apex with an accuracy of $0.001 \mathrm{~mm}$ and a magnification of 6 (Figure 4). On the periapical radiograph in the bucco-lingual direction, the difference between the mesial limit of the before instrumentation canal $\left(M_{b}^{P}\right)$ and the mesial limit of the after instrumentation canal $\left(M_{a}^{P}\right)$, and the difference between the distal limit of the before instrumentation canal $\left(D_{b}^{P}\right)$ and the distal limit of the after instrumentation canal $\left(D_{b}^{P}\right)$ were measured and calculated. On the periapical radiograph in the mesio-distal direction, the 
difference between the buccal limit of the before instrumentation canal $\left(B_{b}^{P}\right)$ and the buccal limit of the after instrumented canal $\left(B_{a}^{P}\right)$, and the difference between the lingual limit of the before-instrumentation canal $\left(L_{b}^{P}\right)$ and the lingual limit of the after instrumentation canal $\left(L_{b}^{P}\right)$, were measured and calculated.

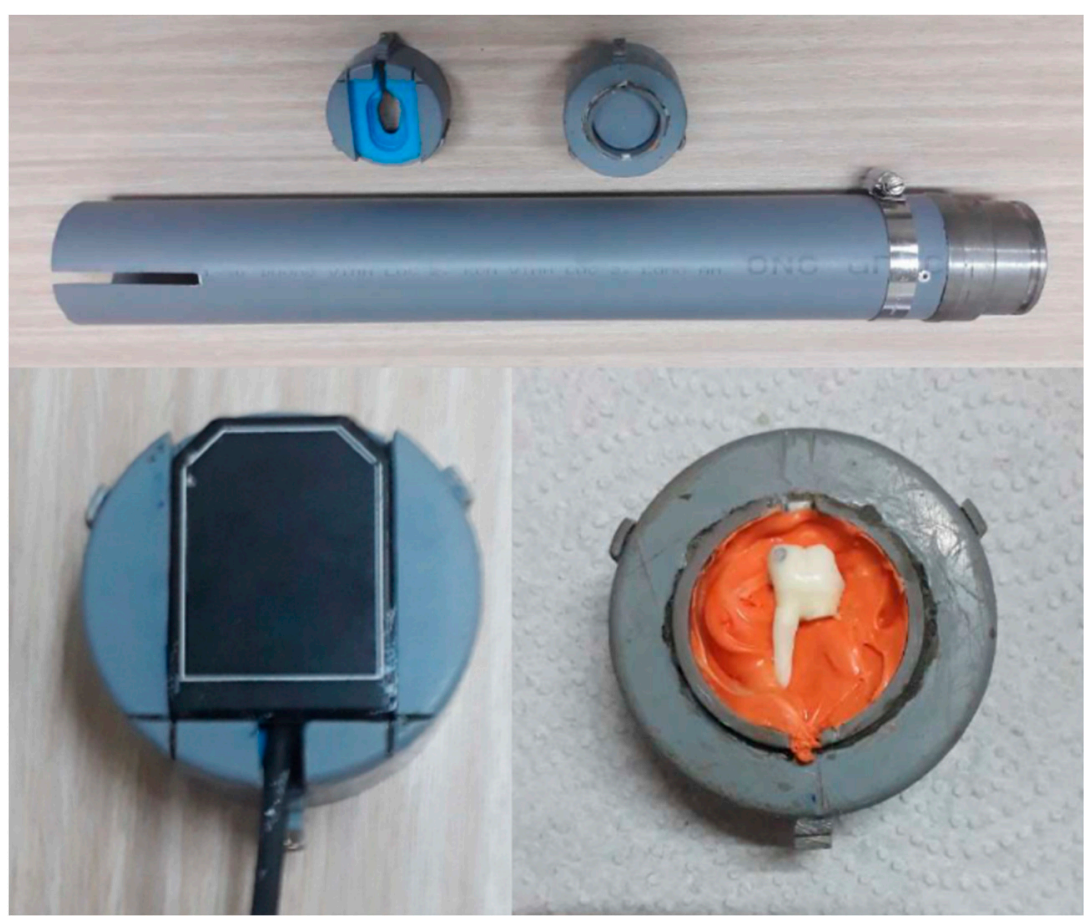

Figure 1. The device for periapical digital radiograph capture. Upper top: parts of the device with long plastic cone at the bottom; lower left: digital sensor in place; lower right: tooth with silicone impression in place.

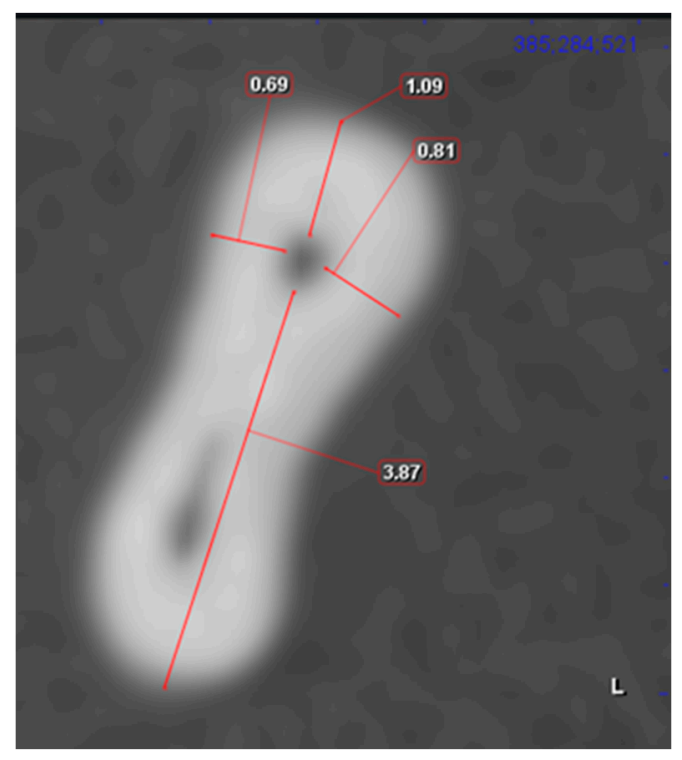

Figure 2. Measurements on CBCT scan. 


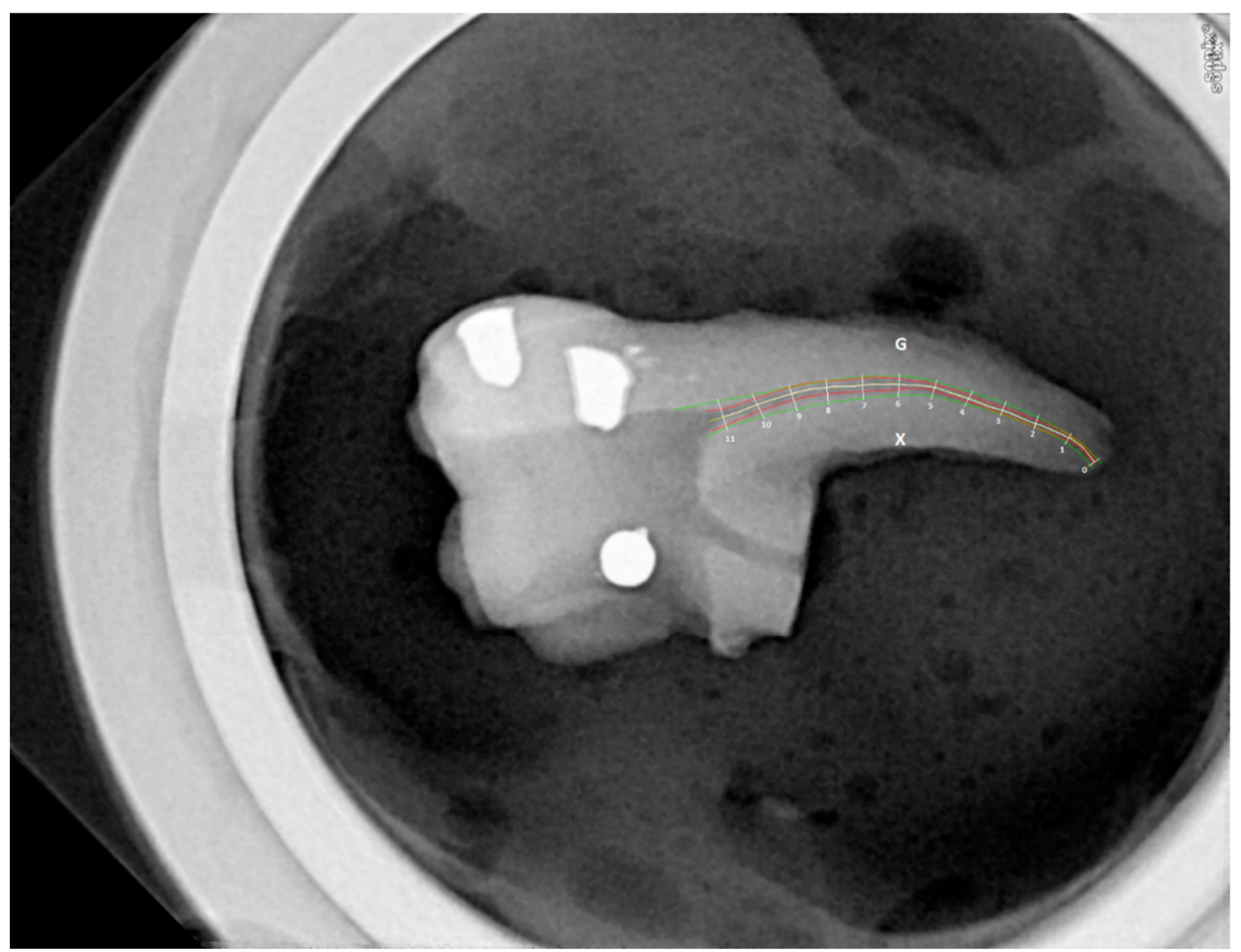

Figure 3. Composite image showing the before instrumentation and after instrumentation tracings.

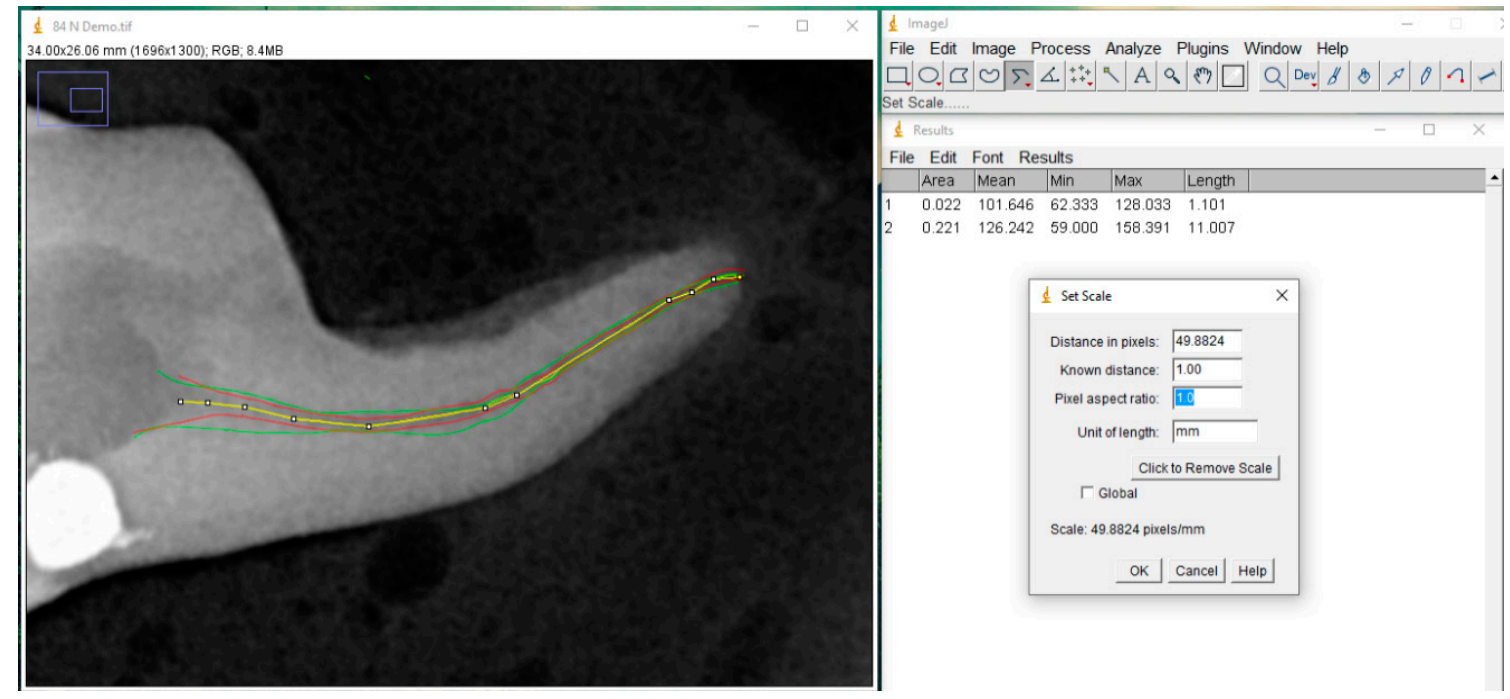

Figure 4. Measurements on periapical digital radiograph image.

All measurements were performed by an endodontist with special training in CBCT scans and related software programs. Intra-class correlation indices were calculated for the examiner four weeks after the first measurements with both CBCT and the periapical radiograph.

The Bland-Altman method was used for detecting the differences between two evaluation methods and the agreement between them using the MedCalc Statistic Software version 19. 


\section{Results}

The ICC indices for the examiner were greater than 0.96 for all measurements in both CBCT scans and periapical radiographs.

The mean bias, confidence intervals, $p$ values in the two statistical tests, fixed or proportional bias at each level for the transportations and centering ratios of the two methods' measurements are displayed in the Table 1 (in mesio-distal direction) and Table 2 (in bucco-lingual direction). These values for all levels of the two methods' measurements are displayed in the Table 3 for both investigated directions.

Table 1. Mean bias, confidence intervals, $p$ values in two statistical tests, fixed or proportional bias at each level for the transportations and centering ratios of the two methods' measurements in the mesio-distal direction.

\begin{tabular}{|c|c|c|c|c|c|c|c|}
\hline \multirow{2}{*}{ Parameters } & \multirow{2}{*}{ Levels (mm) } & \multicolumn{3}{|c|}{ Paired $t$-Test } & \multirow{2}{*}{$\begin{array}{c}\text { Linear Regression } \\
p\end{array}$} & \multirow[t]{2}{*}{ Fixed Bias } & \multirow{2}{*}{ Proportional Bias } \\
\hline & & Mean Bias & $95 \% \mathrm{CI}$ & $p$ & & & \\
\hline \multirow{5}{*}{ Transportation } & 3 & -0.01489 & -0.1162 to 0.08643 & 0.7434 & 0.1974 & No & No \\
\hline & 4 & 0.06433 & -0.04719 to 0.1759 & 0.2201 & $0.0056^{*}$ & No & Yes \\
\hline & 5 & 0.06322 & -0.05968 to 0.1861 & 0.2696 & 0.7004 & No & No \\
\hline & 6 & 0.03778 & -0.06980 to 0.1454 & 0.4415 & 0.6484 & No & No \\
\hline & 7 & -0.07056 & -0.1506 to 0.009513 & 0.0766 & 0.9111 & No & No \\
\hline \multirow{5}{*}{ Centering ratio } & 3 & 0.3951 & -1.2221 to 2.0123 & 0.5886 & 0.8721 & No & No \\
\hline & 4 & 0.6271 & -0.2147 to 1.4689 & 0.1241 & 0.0114 * & No & Yes \\
\hline & 5 & 0.1771 & -1.7706 to 2.1247 & 0.8392 & 0.0527 & No & No \\
\hline & 6 & 0.3352 & -0.2695 to 0.9399 & 0.2370 & 0.2078 & No & No \\
\hline & 7 & -0.3834 & -0.7697 to 0.002949 & 0.0514 & 0.2339 & No & No \\
\hline
\end{tabular}

${ }^{*}$ Differences at a significant level of 0.05 .

Table 2. Mean bias, confidence intervals, $p$ values in two statistical tests, fixed or proportional bias at each level for the transportations and centering ratios of the two methods' measurements in the bucco-lingual direction.

\begin{tabular}{|c|c|c|c|c|c|c|c|}
\hline \multirow{2}{*}{ Parameters } & \multirow{2}{*}{ Levels (mm) } & \multicolumn{3}{|c|}{ Paired $t$-Test } & \multirow{2}{*}{$\begin{array}{c}\text { Linear Regression } \\
p\end{array}$} & \multirow[t]{2}{*}{ Fixed Bias } & \multirow{2}{*}{ Proportional Bias } \\
\hline & & Mean Bias & $95 \%$ CI & $p$ & & & \\
\hline \multirow{5}{*}{ Transportation } & 3 & -0.01700 & -0.07900 to 0.04500 & 0.5448 & 0.6202 & No & No \\
\hline & 4 & 0.002000 & -0.1571 to 0.1611 & 0.9776 & 0.2485 & No & No \\
\hline & 5 & -0.05544 & -0.1687 to 0.05778 & 0.2915 & 0.0978 & No & No \\
\hline & 6 & 0.01144 & -0.2180 to 0.2408 & 0.9112 & 0.7939 & No & No \\
\hline & 7 & -0.01744 & -0.2471 to 0.2122 & 0.8653 & $0.0211^{*}$ & No & Yes \\
\hline \multirow{5}{*}{ Centering ratio } & 3 & 0.7195 & -0.4977 to 1.9368 & 0.2100 & 0.2946 & No & No \\
\hline & 4 & -0.5300 & -2.1093 to 1.0492 & 0.4612 & $0.0076^{*}$ & No & Yes \\
\hline & 5 & -0.1309 & -2.8404 to 2.5786 & 0.9140 & 0.3154 & No & No \\
\hline & 6 & -0.1871 & -4.0681 to 3.6940 & 0.9142 & 0.9227 & No & No \\
\hline & 7 & -1.2193 & -3.7273 to 1.2887 & 0.2948 & 0.0011 * & No & Yes \\
\hline
\end{tabular}

* Differences at a significant level of 0.05 .

Table 3. Mean bias, confidence intervals, $p$ values in two statistical tests, fixed or proportional bias at all five levels for the transportations and centering ratios of the two methods' measurements in both directions.

\begin{tabular}{|c|c|c|c|c|c|c|c|}
\hline \multirow{2}{*}{ Directions } & \multirow{2}{*}{ Parameters } & \multicolumn{3}{|c|}{ Paired $t$-Test } & \multirow{2}{*}{$\begin{array}{c}\text { Linear Regression } \\
p p\end{array}$} & \multirow[t]{2}{*}{ Fixed Bias } & \multirow{2}{*}{ Proportional Bias } \\
\hline & & Mean Bias & $95 \%$ CI & $p$ & & & \\
\hline \multirow{2}{*}{ Mesio-distal } & Transportation & 0.01598 & -0.02643 to 0.05839 & 0.4517 & 0.1612 & No & No \\
\hline & Centering ratio & 0.2302 & -0.2416 to 0.7020 & 0.3308 & 0.8432 & No & No \\
\hline \multirow{2}{*}{ Bucco-lingual } & Transportation & -0.01529 & -0.07964 to 0.04906 & 0.6344 & 0.2332 & No & No \\
\hline & Centering ratio & -0.2696 & -1.2411 to 0.7020 & 0.5789 & 0.3601 & No & No \\
\hline
\end{tabular}


In the mesio-distal direction, there was not any fixed bias at all levels for the two parameters and there were two proportional biases at the level of $4 \mathrm{~mm}$ for the two parameters; therefore, there was an agreement between the two methods' measurements.

In the bucco-lingual direction, there was not any fixed bias at all levels for the two parameters and there were three proportional biases at the level of $4 \mathrm{~mm}$ and $7 \mathrm{~mm}$ for the two parameters; therefore, there was an agreement between the two methods' measurements.

For both the mesio-distal and bucco-lingual directions, there was not any fixed or proportional bias on the whole investigated root canal length of $4 \mathrm{~mm}$ (from a level of $3 \mathrm{~mm}$ to $7 \mathrm{~mm}$ ) for the two parameters; there was an agreement between the two methods' measurements.

The Bland-Altman plots for the agreements between the two methods' measurements are displayed in the four figures, Figures 5-8.

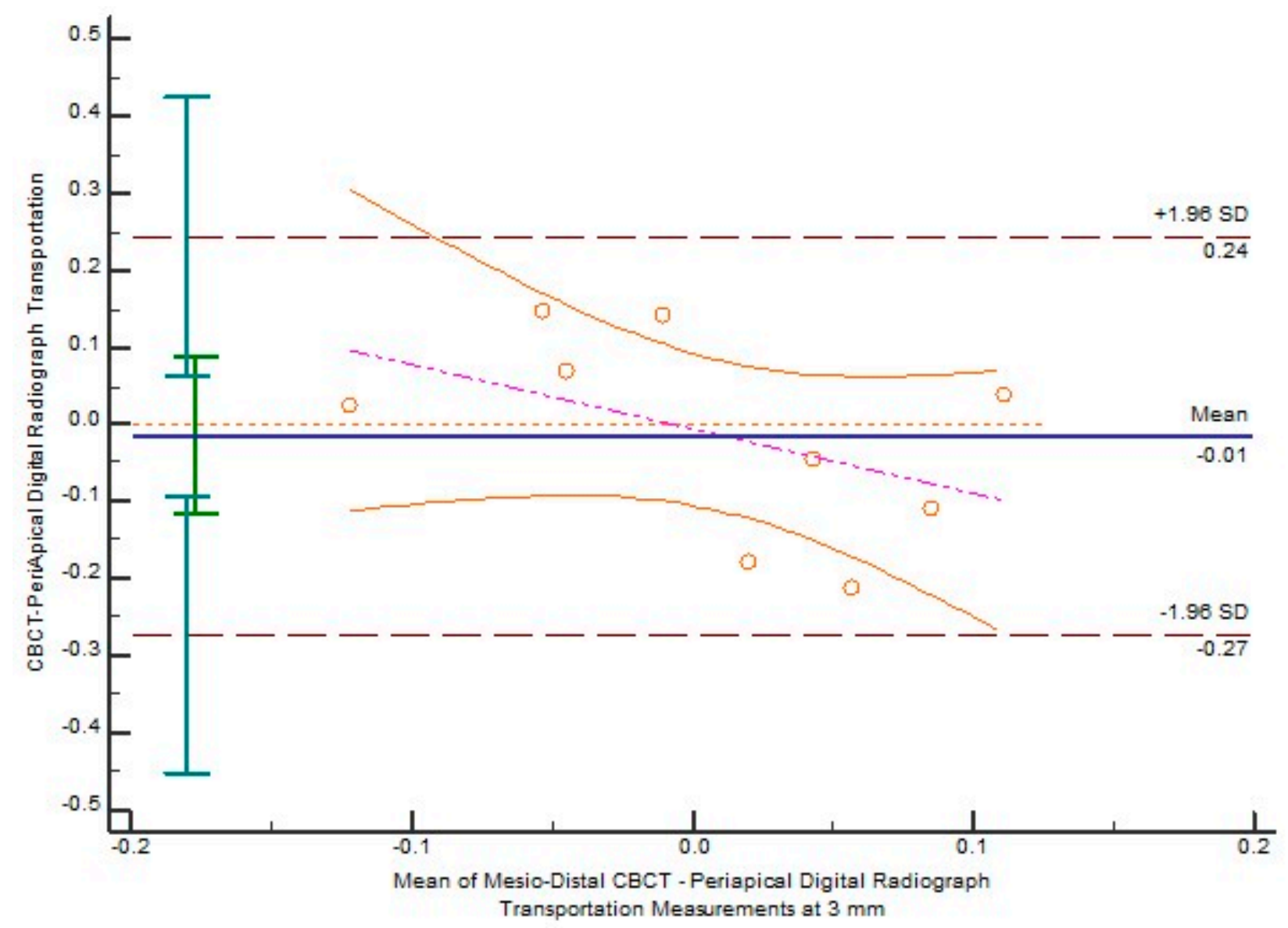

Figure 5. Bland-Altman plot for the agreement between the two methods' measurements for the transportation in the mesio-distal direction, at a level of $3 \mathrm{~mm}$. 


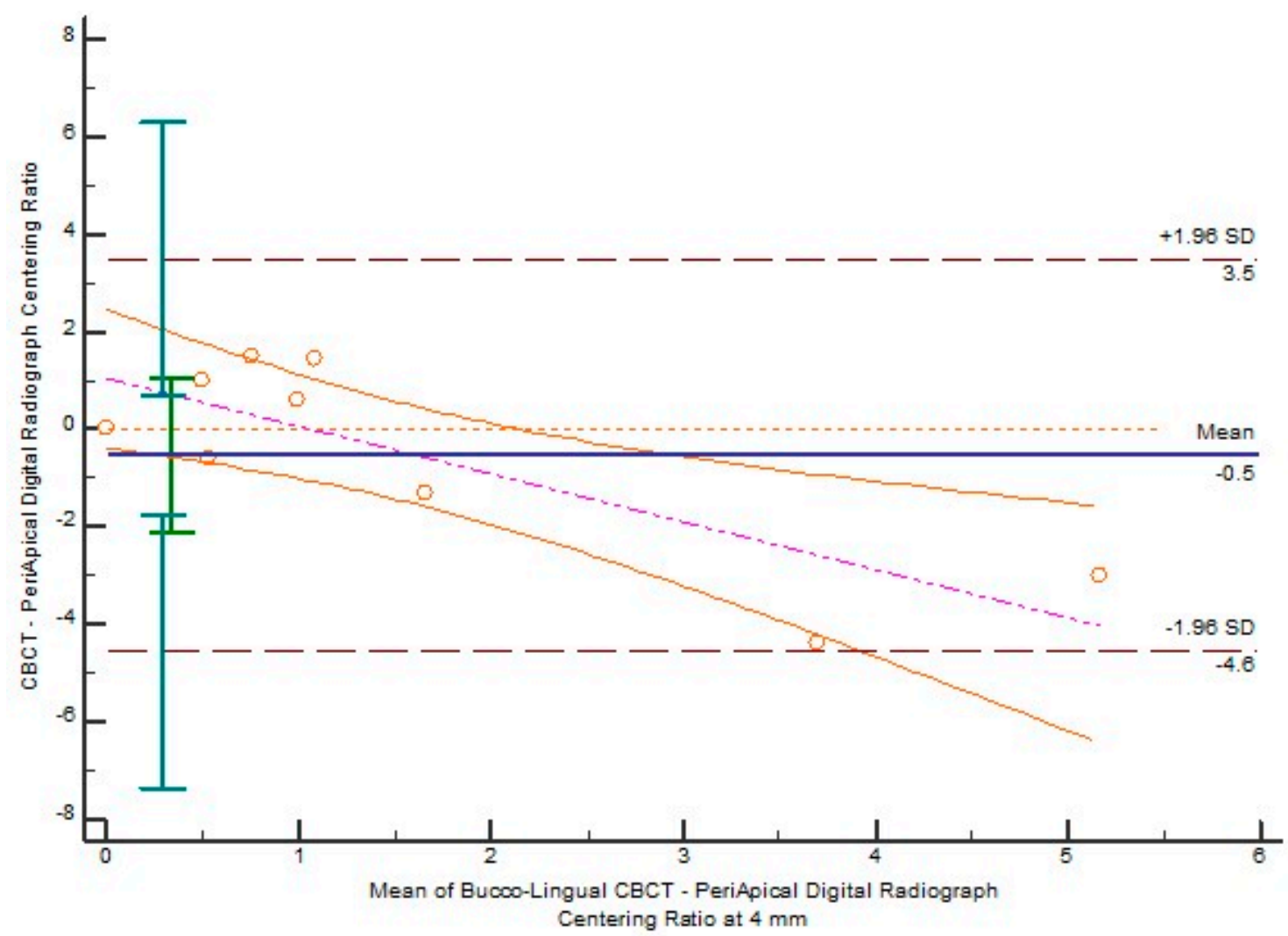

Figure 6. Bland-Altman plot for the agreement between the two methods' measurements for the centering ratio in the bucco-lingual direction, at a level of $4 \mathrm{~mm}$.

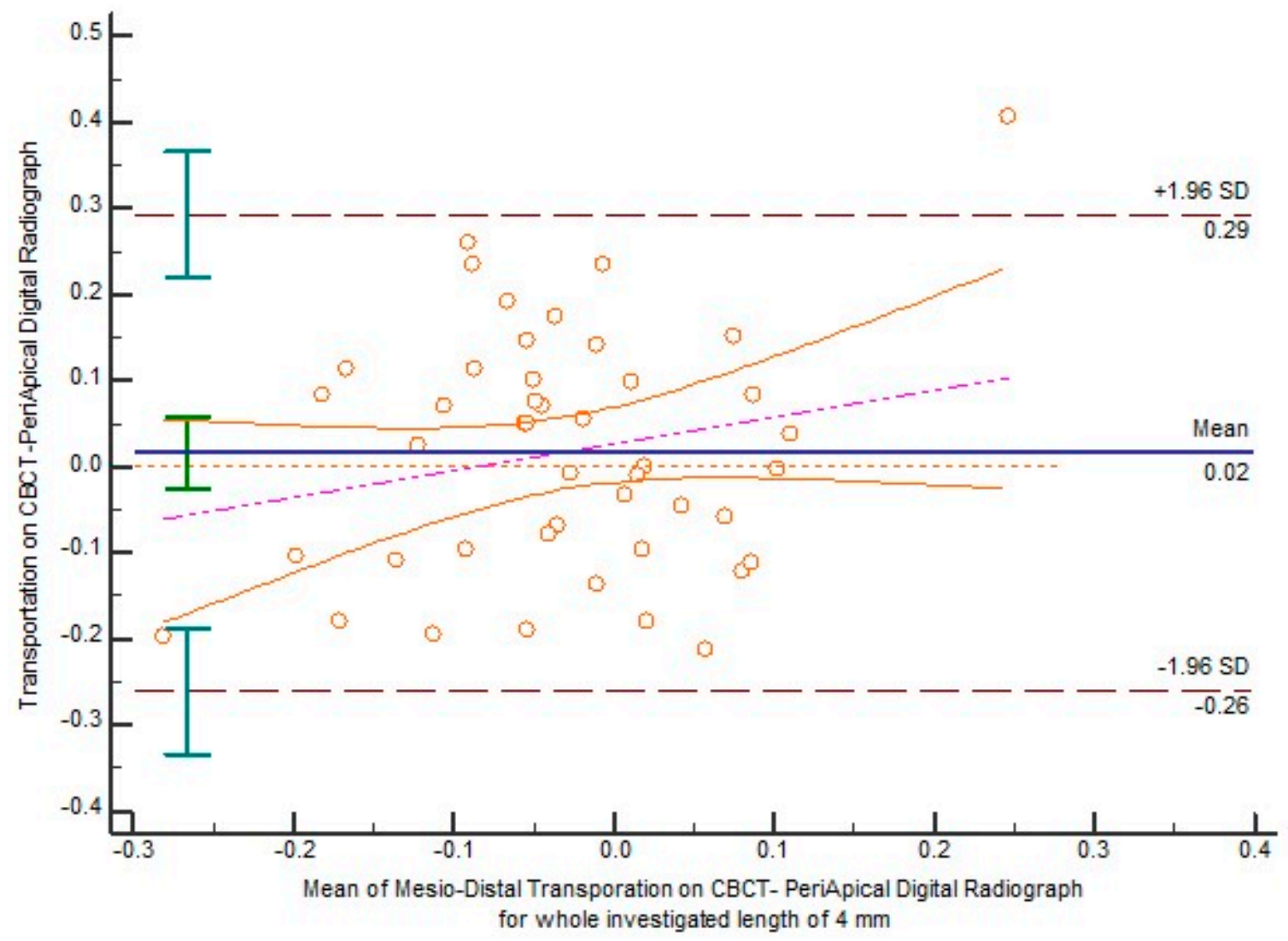

Figure 7. Bland-Altman plot for the agreement between the two methods' measurements for the transportation in the mesio-distal direction, for the whole investigated length of $4 \mathrm{~mm}$. 


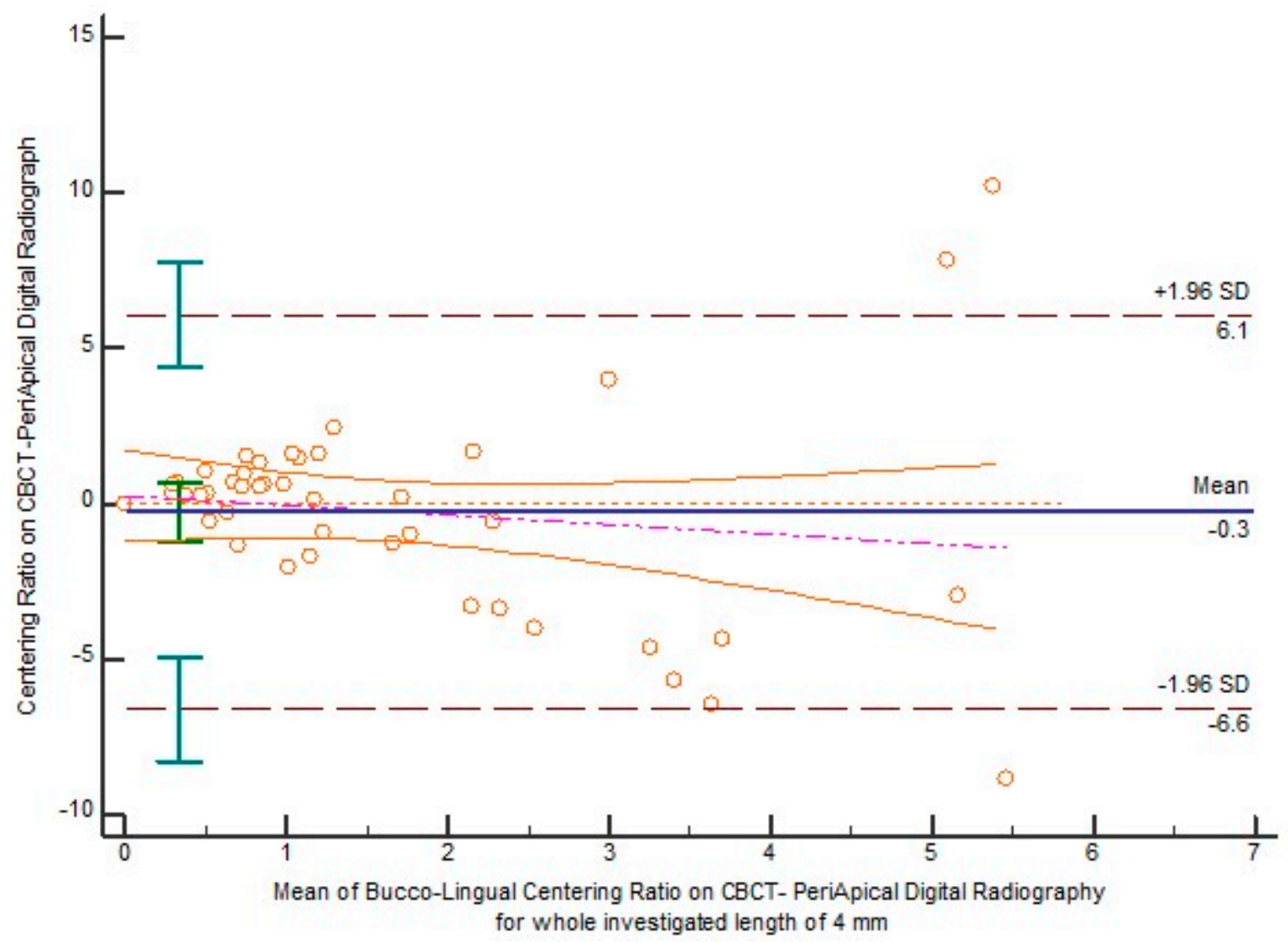

Figure 8. Bland-Altman plot for the agreement between the two methods' measurements for the centering ratio in the bucco-lingual direction, for the whole investigated length of $4 \mathrm{~mm}$.

\section{Discussion}

The evaluation of root canal transportation using different modalities on extracted human teeth was one of the most popular methods in endodontic preparation research [4]. Among the methods, the radiograph was used commonly because of its non-destructive characteristics, avoiding biases resulting from the invasive manipulation of the specimen [4].

One of the most critical factors when manipulating subtraction or imaging overlapping techniques in periapical radiographs is the acquisition of the same position of the specimen both before and after intervention. Registration impressions of putty silicone and accurate removable gear are popularly used for this purpose. The requirement for three radiopaque marks by composite or amalgam on the remaining tooth structure is necessary to ensure the two positions are completely coincided. The long cone technique should be used to obtain the parallel of the ray beam and, therefore, the image dimensions are considered as the actual lengths. An advantage of the periapical radiograph is the production of clearer, more user-friendly images when compared with CBCT. However, this method requires more time and carefulness with the insertion of the specimen into the registration impression and there is a high ratio of discard once there is a mistake.

For the non-clinical or mesio-distal orientation periapical radiography, in the severe curvature canal, there are many overlapping structures of the apical region and the measurements become inaccurate. Therefore, these parameters are referential values, not exact ones for further analysis.

The CBCT modality requires a relative correspondence between the two before and after positions of the specimen because of the flexibility of software special tools in the adjustments of axial, cross-sectional, sagittal slices and other functions. Equipped with 
strong algorithms, CBCT scans possess the actual dimensions, facilitating all measurements at a highly accurate level. Recordation of marks and coordinates for critical special locations ensures that the repetition of all measurements is possible and reliable.

Both the modalities in the present study are non-destructive interventions, opening up a prospective future for in vivo studies should the ethical requirements be satisfied. They require the complete, adequate, and qualitative training of the endodontist or radiologist, who acquire the measurements. These two methods do not need additional enhanced contrast medium inside the canal like other methods [13], ensuring the clarity of images and the accuracy of measurements.

The measurement on the periapical radiograph for the evaluation of pre-instrumentation root dentin thickness was used in a previous study with a certain success [2].

The smallest voxel size of the CBCT is $0.075 \mathrm{~mm}$, far larger than that of micro-CBCT, leading to less accuracy of the measurements. This is also the main shortcoming of this modality as it could not gain the measurements at $2 \mathrm{~mm}$ from the apex of the root [1].

Radiation scatter is another drawback of evaluation using CBCT, making measurements of certain configurations impossible in teeth with heavy metal restoration. However, as to the apical region with horizontal slices, the measurement is not affected by the radiation scatter from the coronal restoration. In the present study, there is only a slight effect of the three amalgam cavities on the coronal part of the tooth, which influences the most coronal cross-sectional slice.

In the present study, CBCT seems to be the time saving modality when compared with the periapical digital radiograph because of the batch capture of five or six specimens at once.

One of the most common parameters used for the agreement between two methods for measuring a quantitative variable was the intraclass correlation index with the lower bound of 0.75 [14]. However, this concept was opposed by the following study with another approach to solve the problem [15].

The comparison of means from different measurements was commonly used for the evaluation of accuracy between different methods $[3,11]$. The results from these two studies revealed that there were significant differences between the means from different methods of evaluation, preventing further comparisons among the methods $[3,11]$. The result of the present study revealed that there was an agreement between both methods using periapical digital radiography and CBCT in the evaluation of root canal transportation with the same levels of investigation. These two evaluation methods could be used interchangeably under the conditions of the present study.

Previously, a Bland-Altman plot was commonly used for the evaluation of endodontic length measurements using different modalities with certain limited effectiveness [7]. Recently, the Bland-Altman method, including appropriate tests and plots, revealed its usefulness in this kind of evaluation $[5,6,8]$.

The Bland-Altman method with appropriate statistical tests and plots proves its justifiable rationale in this case. Just the correlation between measurements of the two modalities is not enough to explain and justify the accuracy of the one versus the other [16-18]. The agreement between the two methods is the prerequisite for further application of both measurements in future studies [16-18]. The comparisons of means are not appropriate for this case and a further deeper analysis produces better outcomes [16-18].

In the present condition of an in vitro setting, with a minimum of artifacts because of any radiation scatter from other surrounding structures, the measurements could be obtained at the $2 \mathrm{~mm}$ level from the end of preparation using CBCT scans. This might not be reasonable for clinical settings, where the surrounding structures are the main obstructions to clear images, as proved by a previous study [19]. This is an in vitro study in which the direction and position of root and X-ray can be standardized; however, in a clinical setting, the supporting tissues around a tooth, X-ray film deformation in the mouth and the $X$-ray cone direction may vary between operators and cause distortion of the images. 


\section{Conclusions}

The two methods could be used interchangeably for the measurements of transportation and calculating centering ratio.

Funding: This research received no external funding.

Institutional Review Board Statement: The present study was approved by the Research Ethics Committee of the University of Medicine and Pharmacy at Ho Chi Minh City, Vietnam, with the approval number of 353/QĐ-ĐHYD-RHM. The study was performed in accordance with relevant guidelines and regulations. The study acquired the intact human extracted molars obtained from many hospitals for many reasons, with the informed consents were obtained from all participants.

Informed Consent Statement: Informed consent was obtained from all subjects involved in the study.

Data Availability Statement: The datasets used and/or analyzed during the current study are available from the corresponding author on reasonable request.

Conflicts of Interest: The author declares no conflict of interest.

\section{References}

1. Pham, K.; Phan, T. Evaluation of root canal preparation using two nickel-titanium instrument systems via cone-beam computed tomography. Saudi Endod. J. 2019, 9, 210-215. [CrossRef]

2. Pham, K.; Nguyen, N. Cutting efficiency and dentinal defects using two single-file continuous rotary nickel-titanium instruments. Saudi Endod. J. 2020, 10, 56-60. [CrossRef]

3. Freire, L.G.; Gavini, G.; Cunha, R.S.; Santos, M.D. Assessing apical transportation in curved canals: Comparison between cross-sections and micro-computed tomography. Braz. Oral Res. 2012, 26, 222-227. [CrossRef] [PubMed]

4. Fidler, A.; Plotino, G.; Kuralt, M. A critical review of methods for quantitative evaluation of root canal transportation. J. Endod. 2021, 47, 721-731. [CrossRef] [PubMed]

5. Van Pham, K. Endodontic length measurements using 3D Endo, cone-beam computed tomography, and electronic apex locator. BMC Oral Health 2021, 21, 271. [CrossRef] [PubMed]

6. Van Pham, K. Endodontic length measurements using cone beam computed tomography with dedicated or conventional software at different voxel sizes. Sci. Rep. 2021, 11, 9432. [CrossRef] [PubMed]

7. Pham, K.V.; Khuc, N.K. The accuracy of endodontic length measurement using cone-beam computed tomography in comparison with electronic apex locators. Iran. Endod. J. 2020, 15, 12-17. [CrossRef]

8. Nguyen, P.; Pham, K. Endodontic length measurements using different modalities: An in vitro study. J. Int. Soc. Prev. Community Dent. 2020, 10, 752-758. [CrossRef] [PubMed]

9. Portelli, M.; Militi, A.; Lo Giudice, A.; Lo Giudice, R.; Rustico, L.; Fastuca, R.; Nucera, R. 3D assessment of endodontic lesions with a low-dose CBCT protocol. Dent. J. 2020, 8, 51. [CrossRef] [PubMed]

10. Lo Giudice, R.; Nicita, F.; Puleio, F.; Alibrandi, A.; Cervino, G.; Lizio, A.S.; Pantaleo, G. Accuracy of periapical radiography and CBCT in endodontic evaluation. Int. J. Dent. 2018, 2018, 2514243. [CrossRef] [PubMed]

11. Ammar, S.A.; Sabet, N.E.; Bastawy, H.A. Accuracy of CBCT, digital and conventional radiography in evaluating the shaping performance of a novel niti rotary system (an in vitro study). Egypt. Dent. J. 2020, 66, 2751-2761. [CrossRef]

12. Schneider, S.W. A comparison of canal preparations in straight and curved root canals. Oral Surg. Oral Med. Oral Pathol. 1971, 32, 271-275. [CrossRef]

13. Neelakantan, P.; Subbarao, C.; Subbarao, C.V. Comparative evaluation of modified canal staining and clearing technique, cone-beam computed tomography, peripheral quantitative computed tomography, spiral computed tomography, and plain and contrast medium-enhanced digital radiography in studying root canal morphology. J. Endod. 2010, 36, 1547-1551. [CrossRef] [PubMed]

14. Lee, J.; Koh, D.; Ong, C.N. Statistical evaluation of agreement between two methods for measuring a quantitative variable. Comput. Biol. Med. 1989, 19, 61-70. [CrossRef]

15. Bland, J.M.; Altman, D.G. A note on the use of the intraclass correlation coefficient in the evaluation of agreement between two methods of measurement. Comput. Biol. Med. 1990, 20, 337-340. [CrossRef]

16. Bland, J.M.; Altman, D.G. Measuring agreement in method comparison studies. Stat. Methods Med. Res. 1999, 8, 135-160. [CrossRef] [PubMed]

17. Bland, J.M.; Altman, D.G. Statistical methods for assessing agreement between two methods of clinical measurement. Lancet 1986, 1, 307-310. [CrossRef]

18. Altman, D.G.; Bland, J.M. Measurement in medicine: The analysis of method comparison studies. J. R. Stat. Soc. Ser. D 1983, 32, 307-317. [CrossRef]

19. Bui, A.; Pham, K. Evaluation of reparative dentine bridge formation after direct pulp capping with biodentine. J. Int. Soc. Prev. Community Dent. 2021, 11, 77-82. [CrossRef] [PubMed] 